to dementia service plans; To build collaborative and mutually supportive ways of working with local dementia services; To engage with the community in the development of Dementia Action Alliances.

Conclusions Key learning from this process is that hospices need to be proactive to ensure their service is responsive to people with dementia. Community engagement and cross organisational working is crucial to this with the Hospice being involved in dementia service development and highlighting their commitment to equality of access for people with dementia and their families.

\section{P-135 THE HOSPICE DEMENTIA NURSE FOR CARE HOMES PROJECT}

Janet Willoughby. The Hospice of St Francis, Berkhamsted, UK

\subsection{6/bmjspcare-2016-001245.158}

Context People with dementia may receive sub-optimal end-oflife care (EoLC) in comparison with those who are cognitively intact (Lillyman and Bruce, 2016). Care home staff report feeling poorly-equipped to manage EoLC in dementia (Kupeli et al., 2016). Anecdotal evidence from the delivery of the ABC End of Life Care education programme for care homes by the Hospice of St Francis found challenges for staff included assessment, recognition of dying and symptom control.

The Dementia Nurse in Care Homes project aims to support care home staff in improving the quality of life of people with dementia and their families. A dementia nurse from the Hospice of St Francis will work in three specialist dementia units, a residential care home, a nursing home and a mental health care unit. Objectives

1. Provide clinical support to care home staff to recognise when the person is dying.

2. Promote compassionate, holistic care in the individual's familiar environment, preventing inappropriate hospital admission and support those important to them through the bereavement process.

3. Initiate and support advance care planning, designing and embedding the documentation, culture and education required to sustain this process.

4. Provide EoLC education through the ABC programme, training the staff to use a supportive care register.

Evaluation and sustainability $\mathrm{The} \mathrm{ABC}$ end of life care education programme uses a Palliative and EoLC Checklist for care homes for each patient, to record all aspects of support in place, and measure improvements in care. This will be the outcome tool for the Dementia Nurse project. The project is co-funded by Hospice UK and St James Place; the Hospice of St Francis is committed to continuing the post at the project end.

\section{P-136 ADVANCE CARE PLANNING AND ADVANCE HEALTHCARE DIRECTIVES WITH A PERSON WITH DEMENTIA}

${ }^{1}$ Deirdre Shanagher, ${ }^{1}$ Marie Lynch, ${ }^{1} J o h n$ Weafer, ${ }^{2}$ Willie Molloy, ${ }^{3}$ Ruth Esther Beck, ${ }^{1}$ Patrica Rickard-Clarke, ${ }^{4}$ Sharon Beatty, ${ }^{5}$ Emer Begley. ${ }^{1}$ Irish Hospice Foundation, Dublin, Ireland; ${ }^{2}$ University College Cork and university Hospital Cork; ${ }^{3}$ Ulster University; ${ }^{4}$ University College Hospital Galway; ${ }^{5}$ The Alzheimer Society of Ireland
Background Dementia is a progressive life-limiting illness. People with dementia value planning ahead. It allows them to express wishes and preferences and reduces anxiety. With the enactment of The Assisted Decision Making (Capacity) Act 2015 guidance in relation to advance care planning and advance healthcare directives with people with dementia is required by health and social care professionals.

Methods An expert advisory group was established. A systematic literature review, searching online databases, CINAHL and PubMed was carried out. Grey literature was also accessed. The themes were presided on by the expert advisory group. Identified literature review themes directed the scope of the guidance.

Results 288 articles were deemed appropriate. Post review with the expert advisory group the scope of the guidance document was extended to reach the person with dementia and family carers as well as healthcare staff across all settings.

The themes from the literature include:

1. Advance care planning and advance healthcare directives with people with dementia

a. Advance care planning is difficult to engage in due to fluctuating capacity.

2. Family members:

a. Uncertain about roles in advance care planning and having conversations.

3. Professional uncertainty

a. Time constraints, lack of knowledge and understanding of dementia, advance care planning and legal responsibilities are factors.

Guidance is offered on each on each of the above areas.

Conclusion A guidance document has been prepared for health and social care staff to provide palliative care to people with dementia. The document will be published and made available via the Irish Hospice Foundation website.

\section{P-137 SUPPORTING PEOPLE WITH DEMENTIA TO DIE AT HOME IN IRELAND}

Deirdre Shanagher, Sarah Cronin, Marie Lynch. Irish Hospice Foundation, Dublin, Ireland

10.1136/bmjspcare-2016-001245.160

Background The Irish Hospice Foundation has funded a nightnursing service for people with conditions other than cancer since 2006. The number of people with dementia referred to the service has risen each year since then. The Irish Hospice Foundation carried out an audit of all of the referrals received where people had a diagnosis of dementia between June and December 2015.

Aim The aim was to explore components of care which support a person with dementia who accessed the night nursing service to die at home.

Method Supplementary information from specialist palliative care teams (SPCT) was gathered for 52 dementia referrals to the night-nursing service between May and December 2015.

Results

- The availability of family/friends to provide care to a person with dementia appears to be a key determinant to them staying at home

- Having a supportive GP was important for a large number of the sample.

- The decision to stay at home was made by the person with dementia or their families in over $50 \%$ of the sample 